\title{
COMPUTED TOMOGRAPHY IN AUTOSOMAL DOMINANT POLYCYSTIC KIDNEY DISEASE (ADPKD)
}

Devendra Patil ${ }^{1}$, Ashutosh Chitnis ${ }^{2}$ Hrishikesh Khaire ${ }^{3}$, Rajiv Hira ${ }^{4}$, Pankaj Yadav ${ }^{5}$

${ }_{1}^{1}$ Resident 3 rd Year, Department of Radiodiagnosis, MGM Medical College and Hospital, Navi Mumbai. ${ }^{2}$ Associate Professor, Department of Radiodiagnosis, MGM Medical College and Hospital, Navi Mumbai. ${ }^{3}$ Resident $2^{\text {nd }}$ Year, Department of Radiodiagnosis, MGM Medical College and Hospital, Navi Mumbai. ${ }^{4}$ Resident $2^{\text {nd }}$ Year, Department of Radiodiagnosis, MGM Medical College and Hospital, Navi Mumbai. ${ }^{5}$ Resident $2^{\text {nd }}$ Year, Department of Radiodiagnosis, MGM Medical College and Hospital, Navi Mumbai.

\section{ABSTRACT}

ADPKD is the most common of the hereditary diseases (1:1000). It is a systemic hereditary disorder that is characterized by cyst formation in ductal organs, particularly the kidney and liver, gastrointestinal, cardiovascular and musculoskeletal abnormalities. ${ }^{1}$ We present a case of a 55-year-old female with ADPKD. PKD is characterised by the presence of multiple cysts (Hence, "polycystic") typically in both kidneys; however, $17 \%$ of cases initially present with observable disease in one kidney with most cases progressing to bilateral disease in adulthood. ${ }^{2}$ It is the most common hereditary cause of End-Stage Renal Failure (ESRF). ${ }^{3}$ It accounts for $4-10 \%$ of all cases of ESRF. ${ }^{3}$

\section{KEYWORDS}

Autosomal Dominant Polycystic Kidney Disease (ADPKD), Computed Tomography (CT), Hypertension.

HOW TO CITE THIS ARTICLE: Devendra Patil, Ashutosh Chitnis, Hrishikesh Khaire, Rajiv Hira, Pankaj Yadav. "Computed Tomography in Autosomal Dominant Polycystic Kidney." Journal of Evolution of Medical and Dental Sciences 2015; Vol. 4, Issue 101, December 17; Page: 16707-16708, DOI: 10.14260/jemds/2015/2499

\section{INTRODUCTION}

Although, it has $100 \%$ penetrance, it has variable expression and does not generally produce symptoms until adult life. About 85 percent of cases are due to a dominant gene located on the short arm of chromosome 16 (The ADPKD-1 gene), whereas 5-10 percent of cases are due to an abnormal gene located on the long arm of chromosome 4 (The ADPKD-2 gene), which causes a milder form of the disorder.

There is cystic dilatation of nephrons involving all segments from the proximal convoluted tubule to collecting duct due to epithelial hyperplasia leading to redundancy and predisposing to cyst formation. Most affected individuals present in the fourth or fifth decade. ${ }^{1}$

Following conditions are well recognised as being associated with ADPKD. 4,5,7,6

- Cerebral berry aneurysms.

- Found in $6 \%$ of patients with ADPKD without a family history of aneurysms.

- Found in up to $16 \%$ of patients with ADPKD with a family history.

- Intracranial dolichoectasia: $2-3 \% .^{3}$

- Mitral valve prolapse: up to $25 \%{ }^{4}$

- Hypertension: up to $80 \%$ adults. $^{5}$

- Small bowel diverticula (perhaps). ${ }^{6}$

- Colonic diverticulosis.

- Bicuspid aortic valve.

- Aortic dissection.

- Cysts in other organs.

- Liver: most common, $75 \%$ by age 60 years. ${ }^{3}$

- Ovaries.

- Spleen: $\sim 5 \%$.

Financial or Other, Competing Interest: None.

Submission 04-12-2015, Peer Review 05-12-2015,

Acceptance 10-12-2015, Published 17-12-2015.

Corresponding Author:

Dr. Devendra Patil,

Department of Radiodiagnosis,

MGM Medical College, Kamothe

Navi Mumbai-410209.

E-mail: devendrrpatil@gmail.com

DOI:10.14260/jemds/2015/2499
- Seminal vesicles: $60 \%$ by age 40 years. ${ }^{3}$

- Prostate: $11 \%$.

- Pancreas: $10 \%$ : N.B. pancreatic cysts are more common in von Hippel Lindau disease (vHL).

The clinical presentation is variable and includes

- Dull flank pain of variable severity and time course: most common.

- Abdominal or flank masses.

- Haematuria.

- Hypertension: usually develops at the same time as renal failure.

- Renal functional impairment to renal failure.

The imaging appearances vary with the severity of the disease. Ultrasound demonstrates cysts in the adolescent or young adult, who is usually not yet clinically symptomatic. CT and MRI are more sensitive and frequently show more cysts than US. ${ }^{1}$

\section{CASE PRESENTATION}

In this case report, a 55-year-old female who is a known case of diabetes type II and hypertension for the last 3 years came at the MGM Hospital with the presenting complaint of left flank pain and generalized headache with a family history of diabetes mellitus type II. Clinical and imaging findings are suggestive of polycystic kidney disease.

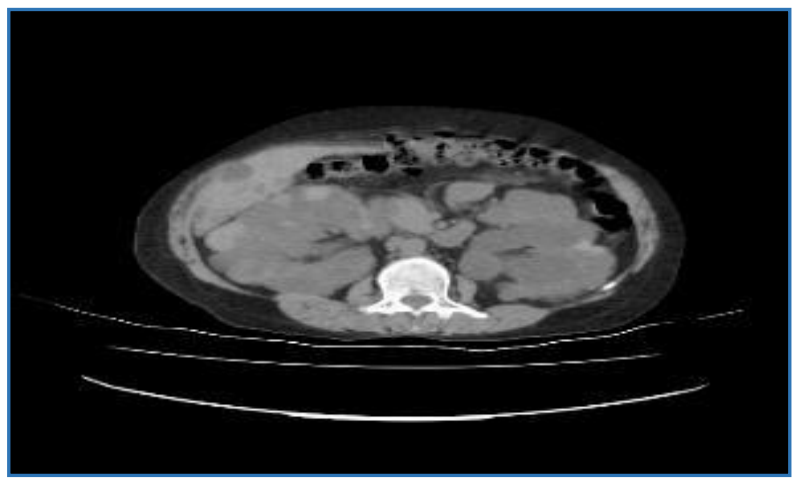

Fig. 1 


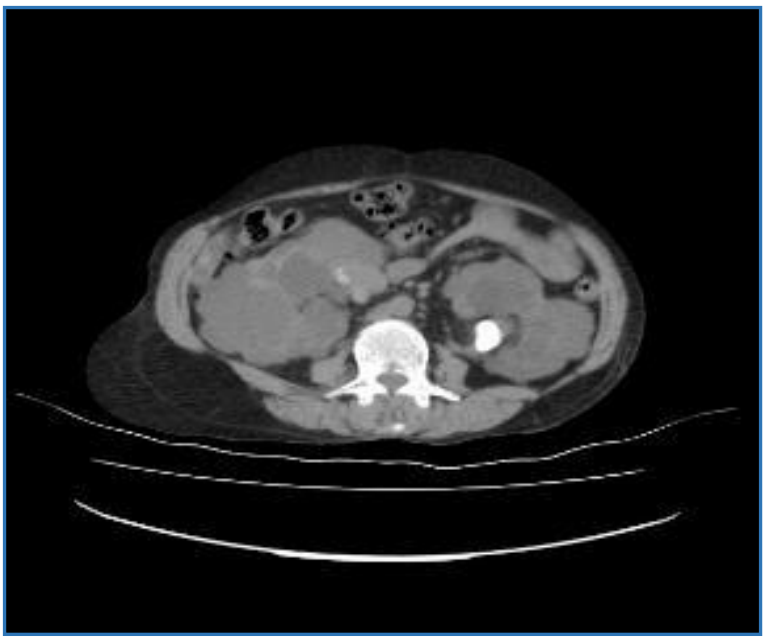

Fig. 2

Fig. 1, 2: Axial non-contrast CT shows multiple cysts in both the kidneys as well as liver with the $20 \mathrm{HU}$ value. A large calculus of about $4 \times 3.2 \mathrm{~cm}$ is seen in the pelvis of left kidney.

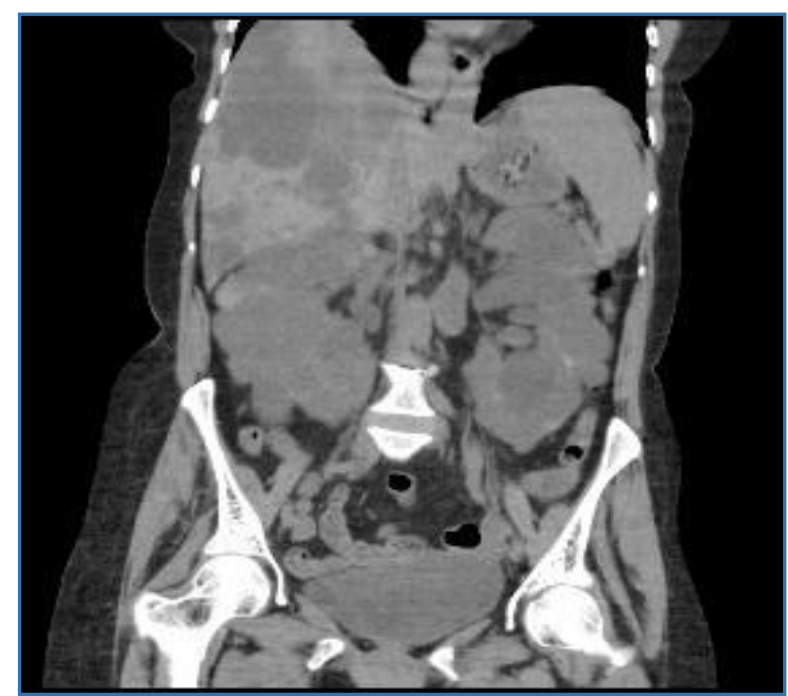

Fig. 3

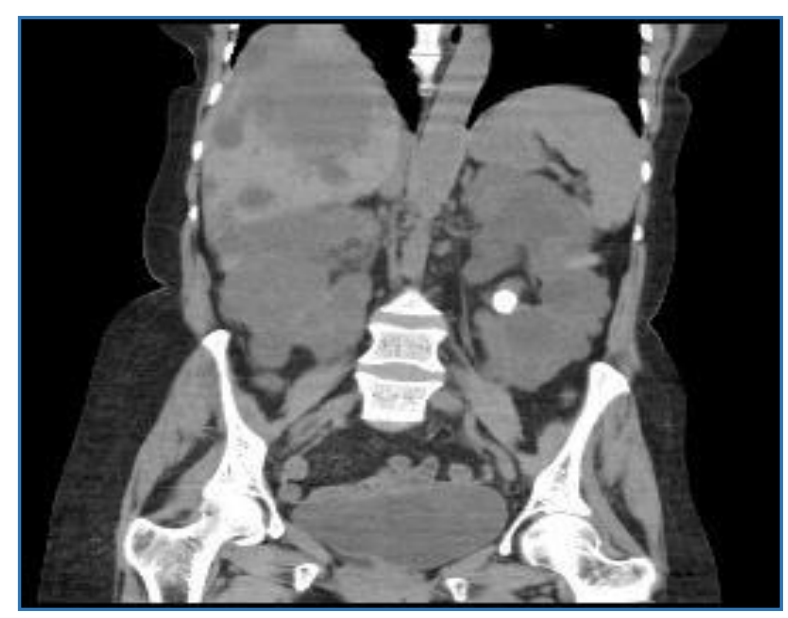

Fig. 4

Fig. 3, 4: Coronal non-contrast CT shows multiple cysts of varying sizes in the liver and kidneys with a left renal calculus. No evidence of cysts are seen within the spleen.

Renal function tests, lipid profile and liver function test were all within normal limits. The patient was managed by
Telmisartan, Amlodipine. This case report illustrates how polycystic kidney disease was diagnosed despite how it presented and being a cause of secondary hypertension.

\section{CONCLUSION}

Adult polycystic kidney disease is an important cause of secondary hypertension in the children and in adults. It can cause various cardiovascular and renal complications, hence making it necessary for this case report.

\section{DISCUSSION}

Adult polycystic kidney disease affects about 12.5 million people worldwide. It is found in all races and affects men and women equally. ${ }^{2}$ The disease affects people of all ages from neonates to adults, for adults usually it effects on in their 50's. It may be life-threatening condition to some individuals, but to some it may be asymptomatic. In our case a 55 years old female was symptomatic, although her blood pressure is currently controlled, but the presence of comorbid diabetes mellitus requires her to have several close monitoring. The average age of end-stage renal disease is approximately 55 years. ${ }^{3}$ Patients with ADPKD may develop Renal Cell Carcinomas (RCC), which typically manifests as atypical renal cysts. Sarcomatous change in these tumours, is thought to be more frequent than in nonADPKD related tumours. ${ }^{2}$

It is usually easy to diagnose Autosomal Dominant Polycystic Kidney Disease (ADPKD) in people who develop flank or abdominal pain and in those who have a family history of ADPKD. An imaging study such as an ultrasound is recommended as the first diagnostic test and may reveal multiple cysts on both kidneys. Cysts may also be seen in the liver, pancreas and spleen. ${ }^{7}$

In people without a family history, ADPKD may be more difficult to diagnose. The diagnosis of polycystic kidney disease may first be suspected based on an imaging test, such as an ultrasound performed for some other reason. The family history may be negative either because family members developed symptoms at a later age and died of other causes before ADPKD was diagnosed.

\section{REFERENCES}

1. Levine E, Hartman DS, Meilstrup JW, et al. Current concepts and controversies in imaging of renal cystic diseases. UCNA 1997;24(3):523-43.

2. Guermazi A. Imaging of kidney cancer. Springer Verlag. (2006) ISBN:3540211292.

3. Morcos SK, Morcos S, Thomsen H. Urogenital Imaging, A Problem-Oriented Approach. Wiley. ISBN:0470510897.

4. Schrier RW. Diseases of the kidney and urinary tract. Lippincott Williams and Wilkins. (2007) ISBN:0781793076.

5. Peña JM, Pernaute R, Vicente de Vera C. Is ADPKD associated with small-bowel diverticular disease? Nephrol Dial Transplant 2000;15(11):1890.

6. Nahm AM, Henriquez DE, Ritz E. Renal cystic disease (ADPKD and ARPKD). Nephrol Dial Transplant 2002;17(2):311-4.

7. Ong AC. Screening for intracranial aneurysms in ADPKD. BMJ. 2009;339(sep212):b3763. 